\title{
A Survey on Real-Time MAC Protocols in Wireless Sensor Networks
}

\author{
Zheng Teng, Ki-Il Kim* \\ Department of Informatics, Research Institute of Computer and Information Communication, \\ Gyeongsang National University, Jinju, Korea \\ E-mail:kikim@gnu.ac.kr \\ Received January 13, 2010; revised February 19, 2010; accepted March 10, 2010
}

\begin{abstract}
As wireless sensor network becomes pervasive, new requirements have been continuously emerged. However, the most of research efforts in wireless sensor network are focused on energy problem since the nodes are usually battery-powered. Among these requirements, real-time communication is one of the big research challenges in wireless sensor networks because most of query messages carry time information. To meet this requirement, recently several real-time medium access control protocols have been proposed for wireless sensor networks in the literature because waiting time to share medium on each node is one of main source for end-to-end delay. In this paper, we first introduce the specific requirement of wireless sensor real-time MAC protocol. Then, a collection of recent wireless sensor real-time MAC protocols are surveyed, classified, and described emphasizing their advantages and disadvantages whenever possible. Finally we present a discussion about the challenges of current wireless sensor real-time MAC protocols in the literature, and show the conclusion in the end.
\end{abstract}

Keywords: Wireless Sensor Networks, Medium Access Control (MAC), Real-Time

\section{Introduction}

A wireless sensor network [1] consists of a large number of small, inexpensive sensor nodes which are distributed over a geographical area for monitoring physical phenomena like temperature, noise, light intensity and speed etc. Traditionally, the largest challenge of sensor network is the limited lifetime because of the battery-powered node [2]. Specially, applications like military operation, factory automation and so on, need a constraint time of a message transmission from source node to destination for guaranteeing validity of the message. For such kind of cases, the real-time system can play a crucial role.

Real-time system is a computing system that must react within precise time constraints to events in the environment. In the real-time computing system, the primary feature is called the deadline, which is the maximum time which it must complete its execution within. In several critical applications, a massage arrived at the destination after the deadline is both late and wrong. The realtime algorithm not only requires low delay of a packet possess but also to meet the deadline, that is the largest difference between real-time sensor networks and con- ventional sensor networks. In fact, whereas the objective of the low delay sensor network is to minimize the average response time of a given set of tasks, the objective of real-time sensor network is to meet the individual timing requirement of each task.

Real-time system provides some important features in the critical applications, including:

- Timeliness-Messages have to be transmitted not only by the time they arrive at the destination but also in the time domain.

- Design for peak load-Real-time systems should not collapse when they encounter a peak-load condition, hence they must be designed to manage all anticipated scenarios.

- Predictability - Real-time system should be able to predict the consequences of any scheduling decision for guaranteeing the performance of applications.

- Fault tolerance-Single failure of transmission should not lead the system to crash. Consequently the real-time systems are designed to be fault tolerant.

- Maintainability-The architecture of a real-time system should be designed to ensure that possible system modifications are easy to perform. 
In order to adapt the energy constrained applications for prolonging the lifetime of sensor network, the proposed medium access control (MAC) protocols primarily focus on reducing energy consumption related to the wireless medium [3]. Therefore, substantial number of the MAC protocols [4-12] for wireless sensor networks are designed in the literature for the traditional challenge: energy-efficiency. Furthermore, other parameters such as latency or throughput are also important for sensor network transmission. However, during the time critical applications, the largest challenge is that how to let the alarm messages meet their deadline for guaranteeing safety of events in the environment. In addition to those applications, energy consumption is the secondary importance just like WSN is employed in natural disaster monitor system.

Another impact of real-time communications is as follows. The traffic load is not regular for monitoring environment by WSN since the environmental conditions constantly change over time. In general the situation of application environment is calm, however, when the emergencies are detected, plenty of information is sensed and needed to be transmitted to the user. The traffic congestion is easier to be triggered since the suddenly increased traffic load. Then some important messages need to wait for a long time to be transmitted to sink even dropped. Under above case, the real-time system has great potential for reliving or avoiding the phenomena which is mentioned above in many applications.

In this paper, we present an introduction to real-time MAC protocols for wireless sensor networks. As realtime MAC sensor networks differ from traditional wireless MAC networks in many points, the primary parameters of them reflect in timing requirement (deadline), energy, multiple flows, etc. All of these characteristics make the traditional wireless MAC protocols not be suitable for real-time sensor networks.

The remainder of this paper is organized as follows. A survey of the proposed real-time MAC protocol for sensor networks is presented in the Section 2. In Section 3, we discuss the challenges of the current real-time MAC protocol for sensor network and show the future work. In Section 5, we make a conclusion.

\section{Real-Time MAC Protocols for Wireless Sensor Network}

In this survey we collect recent real-time MAC protocols proposed in the literature. Several typical protocols are included and discussed in this section. According to the difference of applications, real-time MAC protocol for wireless sensor networks can be classified into two classes: hard real-time MAC protocol and soft real-time MAC protocol.

As it is shown in Figure 1, there is a classification tree for wireless sensor networks real-time MAC protocols. As shown in this taxonomy, in order to guarantee the constraint time for ensuring the validity of alarm messages in the time critical application, several proposed approaches in the literature are classified as branches from hard and soft real-time MAC protocols. In the following section, we present the description of the collection of wireless sensor real-time MAC protocols and evaluate their advantages and disadvantages whenever possible.

\subsection{Hard Real-Time MAC Protocol for Wireless Sensor Networks}

\subsubsection{TDMA Based Real-Time MAC Protocol}

RRMAC [13] is a TDMA based hard real-time MAC protocol for wireless sensor networks. As shown in Figure 2, this protocol proposes a tree structure that the packets could flow continuously from leaf-level nodes to the top-level node. The assignment sequence lets the base station of the top of the tree acquire the data from normal sensor nodes in one superframe duration. Figure 3 shows the superframe structure, which is composed of a beacon only period, contention free period, contention access period, and an inactive period. The protocol uses a beacon frame for synchronizing the sensor network. The nodes adjust their time whenever they receive a beacon frame in the system and then execute the contention free period. The protocol decreases the communication delay by assigning time slots to every node. For multi-hop beacon forwarding, in the beacon only period, each beacon slot is assigned to each coordinator, like the base

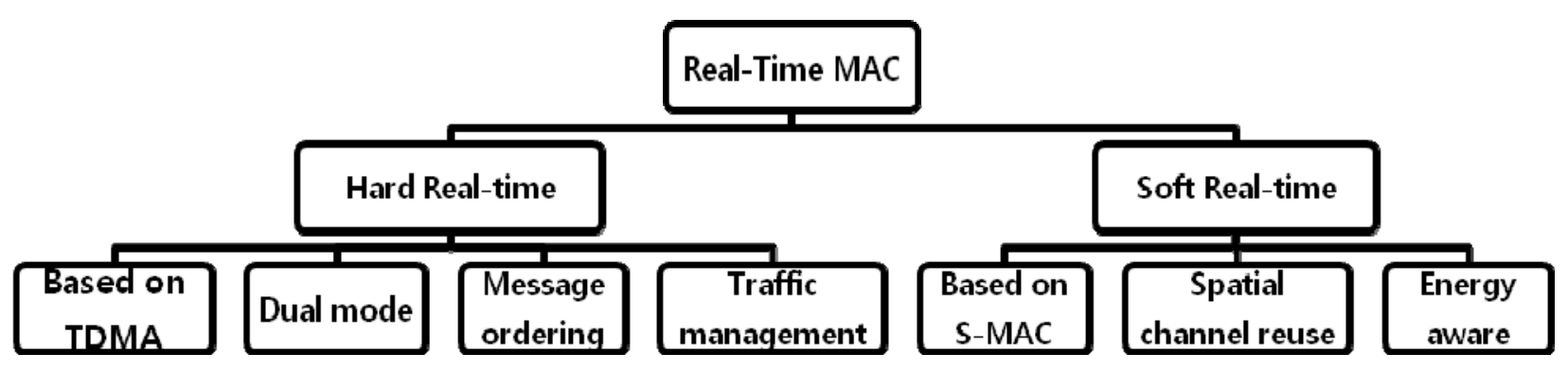

Figure 1. Taxonomy of approaches to real-time MAC protocol in wireless sensor networks 



Figure 2. Time slot assignment for a base station and sink node in RRMAC.

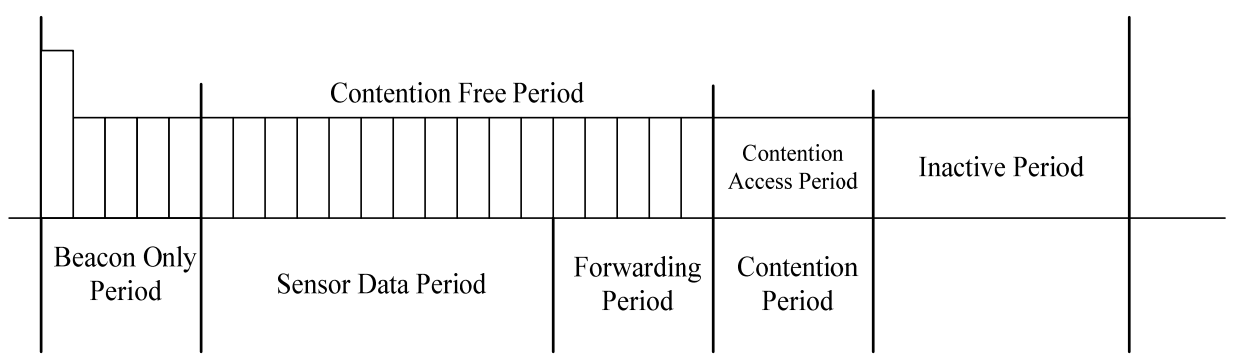

Figure 3. RRMAC superframe structure.

station and sink. This design enables the beacon to be forwarded to the hall network at the beginning of a new superframe.

Several nodes form clusters and each cluster includes a cluster head. RRMAC superframe bases on IEEE 802.15.4 frame structure and only upper level cluster heads can assign time slots in the TDMA superframe. Cluster heads aggregate data collected from lower level sensor nodes and forward the data to upper cluster head in hierarchy. RRMAC nodes are assumed that they have two RF power levels. The sink nodes have high RF power which increases the transmission range of these nodes. Normal nodes have a smaller power and a short communication range. The RRMAC superframe structure is flexible. The superframe can contain only contention period if all of the sensor or sink nodes do not require real time or reliable data transmission. However, the difficulty of RRMAC is maintaining global synchronization in a large randomly distributed multi-hop WSN.

\subsubsection{Two Mode-Based Real-Time MAC Protocols}

Dual-mode real-time MAC protocol $[14,15]$ is hard realtime MAC protocol for wireless sensor networks. This protocol includes two modes, one is protected mode and another is unprotected mode. The Figure 4(a) shows the unprotected mode. In that case, maxrange is the possible max communication range of node. Protocol presents a parameter called backoffunprotected which is the backoff for unprotected mode. When a node sends out an alarm message, nodes that hear its backoffunprotected, which is inversely proportional to their distance to the sending node. A node can be chosen for forwarding the message when no alarm message has been received and backoffunprotected expires. In this way, messages can be transmitted to the sink by a high speed, but not have a high reliability due to collisions. In the protected mode, the protocol provides a high reliability by guaranteeing the collision-free function, however the transmission time is bounded. Every node knows its absolute position since deployment and each message contains the sender's absolute position. In the initial phase, the protocol organizes the network nodes into cells so that all nodes of a cell can communicate with other nodes of two neighboring cells. The unprotected mode does not use cells for transmitting messages. The protected mode uses signaling messages for reserving each cell between source node and sink node (as shown in Figure 4 (b)). Once reserved, a cell cannot generate new messages until the transmission is over for avoiding collision. After initialization, the unprotected mode will first be started but when any node detects a collision, it will send a collided alarm message to another node for switching the mode from unprotected to protected mode. The dual-mode real-time MAC protocol supports the randomly deployed wireless sensor network, and can avoid message collision effectively. However, the protocol requires all the nodes need to know their absolute position information, the assumption is hard to achieve for a randomly deployed WSN. Besides, energy efficiency is not designed in the Dual-mode real-time MAC protocol for sensor network.

\subsubsection{Message Ordering Based Real-Time MAC Protocol}

TOMAC protocol [16] presents hard real-time message ordering at medium access control layer for wireless sensor networks. The hard real-time message ordering mechanism can guarantee the time-order of message delivery in one-hop distance mesh topologies in which the 


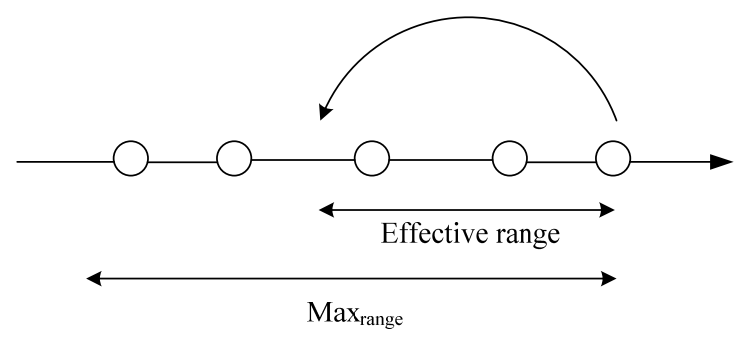

(a) Unprotected mode

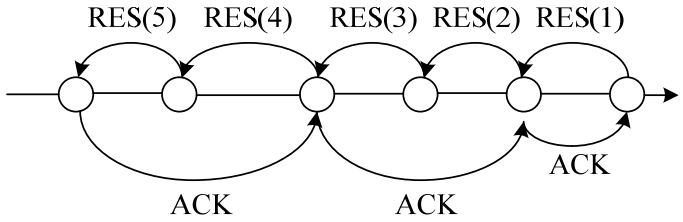

(b) Protected mode

Figure 4. Algorithm of dual-mode real-time MAC protocol.

sensors share the same channel. Ordering messages are based on logical time. For this purpose, each message is assigned a priority, that if a message cannot be transmitted due to the sleep time in a low duty cycle protocol or due to a busy channel, the priority will increase. When the channel is free, the one with the highest priority will gain access. However, TOMAC protocol is difficult to generalize for multi-hop network and other communication topologies.

\subsubsection{Traffic Management Based Real-Time MAC Protocol}

Supporting components for real time sensors (SUPORTS) [17] base on hard real-time at MAC layer for supporting real-time flows in highly unpredictable sensor network environments. The mechanism is based on a joint traffic regulation and end-to-end scheduling approach. This mechanism attempts to maintain accuracy in a resourceefficient manner even under extremely unstable network conditions where delays are difficult to model and compute.

The goal of SUPORTS is to consider the delay requirement of each arriving packet to maximize the probability of meeting its deadline. SUPORTS implements a least-laxity based scheduler component at each sensor node that determines the order with which each individual packet will be delivered by the MAC service. The protocol computes the laxity value $\mathrm{L}$ of a packet as the difference between the deadline and the end-to-end time to transmit the packet from the source to the sink:

$$
L=\text { Deadline }-\left(t_{e l}+t_{s n k}+D\right)
$$

In the equation mentioned above, $t_{e l}$ is the elapsed time since the packet has been initiated at the source and tsnk is the delay that downstream node estimates that will be required until the packet reaches the sink. The $D$ is the local estimation of the projected sojourn time.

In delay sensitive sensor systems the goal of traffic regulation is divided into two parts. First, in cases of congestion, packets need to be dropped to decrease contention and relief overflowing queues in an attempt to reduce delays. Secondly, when a packet is overly delayed, it should not be further forwarded since that would be a waste of transmission energy. Even if the deadline of a packet is large, the packet may still miss its deadline because it might be dropped due to congestion.

\subsection{Soft Real-Time MAC Protocol for Wireless Sensor Networks}

\subsubsection{S-MAC Based Real-Time MAC Protocols} Virtual TDMA for Sensors (VTS) [18] MAC protocol is presented based on soft real-time for WSN applications. VTS protocol is based on sensor-MAC (S-MAC) protocol and provides a Time Division Multiple Access (TDMA) access scheme, in which the number of slots equal to number of nodes in a cell (cluster), the nodes in a cluster will transmit in different time slots. VTS synchronization procedure works as S-MAC, but unlike S-MAC, VTS nodes are only allowed to send data in their captured cycle, a node only sends packets every $N_{c}$ cycle, $N_{c}$ is the length of a superframe (as shown in Figure 5). After a number of network setup cycles, the

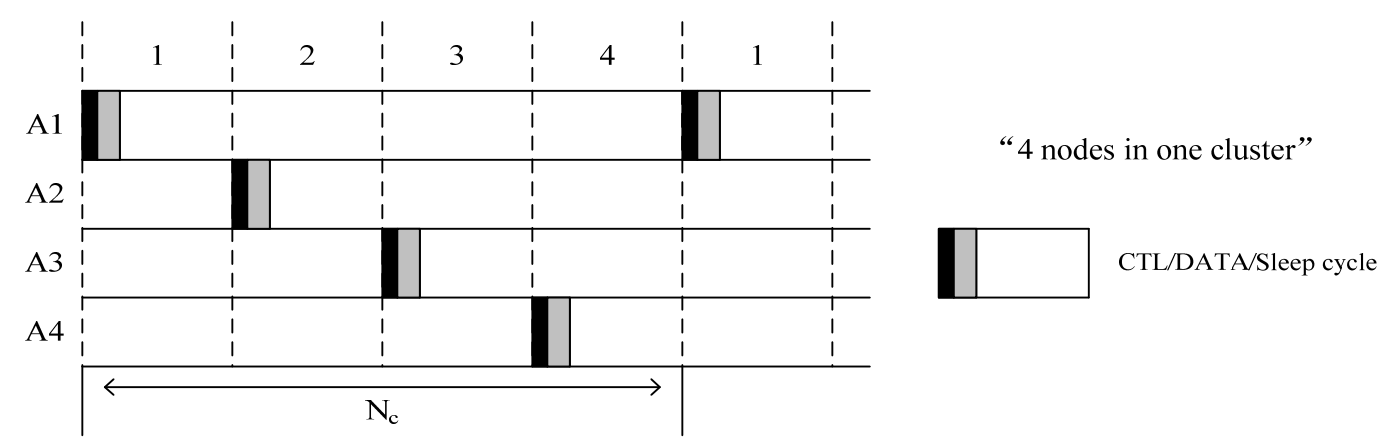

Figure 5. Structure of VTS TDMA frame. 
nodes adjust their superframe length counter to their number of known neighbors.

VTS proposes a control packet called CTL as SYNC packet, when all the nodes have sent their first CTL packet, the virtual superframe of $\mathrm{N}_{\mathrm{c}}$ timeslots is formed. From then on, the node dynamically adapts to the possibility of nodes joining and leaving the cluster by adjusting the length of superframe. VTS uses the CSMA/CA mechanism for data delivery. At the beginning of each timeslot, all the nodes wake up and listen. The owner of the timeslot performs a carrier sense and broadcasts the CTL. The CTL is used as: synchronization schedule discovery, keep-alive beacon, new node discovery, channel reservation. VTS supports three kinds of transmission: unicast packet transmission, broadcast packet transmission and no data transmission. In the unicast packet transmission, the communication has a sequence as $\mathrm{CTL}_{\{\mathrm{RTS}\}} / \mathrm{CTS} / \mathrm{DATA} / \mathrm{ACK}$, both nodes go to sleep after the transmission is finished. During the broadcast packet transmission, a $\mathrm{CTL}_{\{\mathrm{BCAST}\}}$ packet is sent, destination is a broadcast address, without waiting for any CTS reply, sender can send the broadcast packet, after receiving the packet nodes go to sleep and no ACK. When no data transmission, nodes just adjust the clock reference by $\mathrm{CTL}_{\{\mathrm{SYNC}\}}$ packet and go to sleep.

In addition, VTS proposes to dynamically adjust the duty cycle, VTS uses the control center as synchronizer, the sink node controls the synchronization by CTL packet since it is directly connected to the control center.

Compare with S-MAC, VTS decreases energy consumption and the latency of packet transmission when there are only a few nodes. However, when the number of nodes is higher, the energy consumption is also higher. Additional, since the amount of time-slots, it has a limited packet arrival interval, in some cases VTS is very hard to work for a higher packet generation rate.

A novel real-time MAC layer protocol [19] is designed for soft real-time applications in wireless sensor networks. This protocol bases on S-MAC. The novel protocol uses feedback approach as a medium access mechanism. The novel real-time MAC protocol is for single stream communication.

Working of this protocol is based on use of CC control packet which is used to assign an appropriate value to clear channel flag (CCF) of every sensor node. If CCF equals to 1 the nodes can transmit as well as receive data packets, while it can only receive if its CCF value is 0 . Initially all nodes have $\mathrm{CCF}$ value as $1 . \mathrm{CC}$ control packet has a clear channel counter (CCC), its value ranges from 0 to 3 . The value of $\mathrm{CCC}$ is 3 at the originating node of $\mathrm{CC}$ and decreased by one with on hop transmission of $\mathrm{CC}$. CC is always transmitted from sink to source direction. If value of CCC of CC control packet is 2 or 3 in a node, then $\mathrm{CCF}$ of that node will remain 0 , if value of $\mathrm{CCC}$ of $\mathrm{CC}$ is 0 or 1 , the $\mathrm{CCF}$ of that node will become 1 .
Figure 6 explains the novel protocol. As shown in the figure, some data are sent from source node N0 to sink node N9. Duration of one data transfer cycle and one control packet are designated by Tx and Tc respectively. In the first data transfer cycle, RTS/CTS/DATA/ACK will be transmitted by sequence from N0 to N1, after getting ACK, N0 sets its CCF value to 0. From the first duration to fourth duration, the data $\mathrm{P} 0$ is forwarded from N0 to N4 and set its own CCF value to 0. Each packet has a Hop Counter (HC) integer variable whose value varies from 0 to 4 for first 4 hops of a communication stream and 0 to 2 for all later 2 hops segments of the communication stream. At N0, the value of $\mathrm{HC}$ of $\mathrm{P} 0$ is 4 and it is decreased by one each time P0 is transmitted successfully by one hop. Once P0 reaches to N4 node, its $\mathrm{HC}$ becomes 0 . Then $\mathrm{N} 4$ sets $\mathrm{HC}$ of $\mathrm{P} 0$ to 2 . After received ACK from N3, N4 waits for 2Tc duration prior to forwarding P0 to N5. Meantime, in the first and second Tc duration, after receiving $\mathrm{ACK}, \mathrm{N} 3$ and $\mathrm{N} 2$ send $\mathrm{CC}$ signal to $\mathrm{N} 2$ and $\mathrm{N} 1$ respectively. In addition, the CCF of $\mathrm{N} 3$ and N2 are set to 0 . In the next Tc duration, N1 sends $\mathrm{CC}$ signal to N0 and sets its CCF to 1 . Thus, after getting $\mathrm{CC}$ from N1 node, N0 can transmit new packet P1 to N1 in next one Tx duration. After that, $\mathrm{N} 1$ can forward $\mathrm{P} 1$ to $\mathrm{N} 2$ in next Tx duration and wait there for next CC control packet.

Compare with S-MAC and TMAC by the $99 \%$ of duty-cycle, the novel protocol reduces the latency. However, the overhead is higher due to CC control packet. In addition, the novel protocol is difficult to suit to multi-streams communication for WSN.

\subsubsection{Spatial Channel Reuse Based Real-Time MAC Protocol}

Channel Reuse-based Smallest Latest-start-time First (CR-SLF) [20] algorithm schedules messages at MAC layer for increasing spatial channel reuse in soft real-time


Figure 6. The novel real-time MAC protocol frame format. 
multi-hop WSN. This protocol presents an algorithm for mobile wireless sensor network such as a network of mobile robots. The goal of this protocol is to be cognizant of message deadlines at each hop, while avoiding collisions and exploiting spatial reuse. The start time is the time when message is scheduled for transmission and the finish time is the time when the message is completely received by the next hop node. The basic idea is to partition the set of message transmissions into disjoint sets such that transmissions within each set do not interfere with one another and can be executed in parallel.

The algorithm includes three steps. Step 1 selects a transmission to schedule. The scheduler chooses the one with the smallest latest transmission start time (LST), this enables the scheduler to consider the most urgent transmission first. Step 2 assigns this message transmission to a set. The protocol can create $n$ sets: $S_{1}, S_{2}, \ldots, S_{n \text {, }}$ the transmissions in different sets are executed in sequence. The scheduler attempts to assign the transmissions to a suitable set in the set list. Step 3 updates the finish time of the feasible set and insert a new transmission for the next hop. If a feasible set $S_{j}$ is found, then a transmission is inserted into the set, and the new finish time is updated as discussed above.

There is a communication example for describing the algorithm as shown in Figure 7. In that communication, $\mathrm{m}_{1}$ is selected first since the $m_{1}$ has the smallest LST. Then $m_{2}$ is considered since $m_{2}$ has the smallest LST, but $\mathrm{m}_{2}$ interferes with $\mathrm{ml}$, the scheduler can not be set. By calculating the final schedule is set as: $S_{1}=\left\{m_{1}, m_{3}\right\}, S_{2}$ $=\left\{m_{2}\right\}$, which means that $m_{1}$ and $m_{3}$ are transmitted in parallel, followed by the transmission of $m_{2}$.

CR-SLF utilizes a centralized scheduling algorithm, in which the centralized scheduler can decide that when and who will transmit or receive messages. Nevertheless, CR-SLF is not scalable as a centralized scheduling algorithm. Moreover, as a wireless sensor protocol, energy consumption is not provided in [20].

\subsubsection{Energy Based Real-Time MAC Protocols}

Low-power real-time (LPRT) [21] protocol has been proposes at MAC layer for wireless sensing and actuation systems. The LPRT protocol is a hybrid schedule based dynamic TDMA protocol and contention based

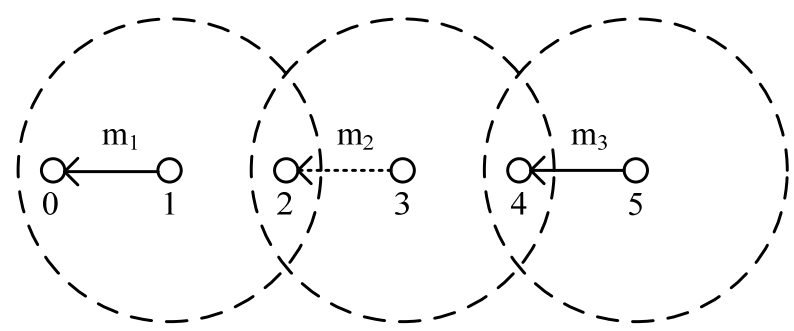

Figure 7. An example for channel reuse in CR-SLF.

CSMA/CA protocol. LPRT considers an infrastructure based star topology, where the stations communicate directly with the base station. If required by the application, the range can be extended with the use of more than one base station, like in a cellular network.

Each superframe of LPRT is divided into a fixed number of mini-slots and starts the transmission by the base station. As shown in Figure 8, the superframe includes beacon frame (B), contention period (CP) and contention free period (CFP). The first one is beacon frame, which is followed by the CP. During the CP any station can transmit packets using CSMA/CA protocol. The CFP is allowed to transmit non-real-time asynchronous traffic if it cannot be completed before the beginning of the CFP. The contention free period is placed after the CP. Transmissions during the CFP are determined by the base station using resource grant $(\mathrm{RG})$ information announced previously in the beacon frame of the current superframe. The CFP is composed by an optional retransmission period (RP) and a normal transmission period (NTP), the retransmission procedure helps to increase the reliability of the protocol.

In LPRT, the station decreases the power consumption and coordinates channel. By using the star topology, there is no overhead related with topology discovery and multi-hop communication. However, the application of LPRT is limited, it's very hard to suit the large multi-hop wireless sensor network and other communication topology.

Asynchronous real-time energy-efficient and adaptive MAC (AREA-MAC) protocol [22] is proposed for supporting real-time and energy efficient applications in wireless sensor networks. AREA-MAC based B-MAC, it reduces latency and energy consumption of nodes by

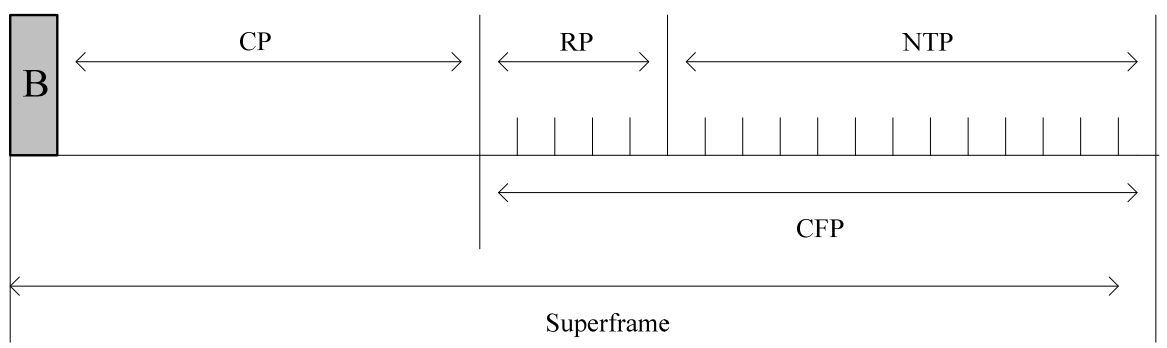

Figure 8. Superframe structure for the LPRT protocol. 
using low power listening (LPL) with short preamble messages, where nodes wake up shortly to check the channel activity, if no packet need to receive or forward packets, they will go back to sleep mode immediately.

The main characteristics of AREA-MAC are asynchrony, energy-efficiency, real-time support and adaptability. For real-time data, the source node requests the suitable next-hop neighbor to wake up regardless to its normal schedule for decreasing the delay. Nodes may change their duty cycle according to the real-time request received from their neighbors. The assumption of topology is a grid-based WSN (as shown in Figure 9), author assumes that the density of nodes is high enough, so that a node can directly communicate with multiple neighbors, nodes are deployed in an order with the sink node having the highest deployment level. Normal nodes forward data only to up-level direction, i.e., towards sink node.

AREA-MAC considers two types of WSN traffic, one is periodic traffic and the other is non-periodic traffic. For periodic traffic, nodes restrict total energy consumption and send data to 1-level, for non-periodic traffic, nodes restrict delay conditions and sent data to 2-level neighbors. For real-time traffic, the sender directly requests its 2-level neighbor to wake up. It further halves latency and saves more energy at 1-level neighbors. However the nodes need more transmission energy, some nodes will die earlier due to energy exhaustion.

\subsection{Real-time MAC Protocol Summary}

This is the summary of several real-time MAC protocols which we presented in this section. For showing the performance, Table 1 is created as a comparison of the realtime MAC protocols.

\section{Open Issues in this Research Field}

In the last section, we presented several real-time MAC

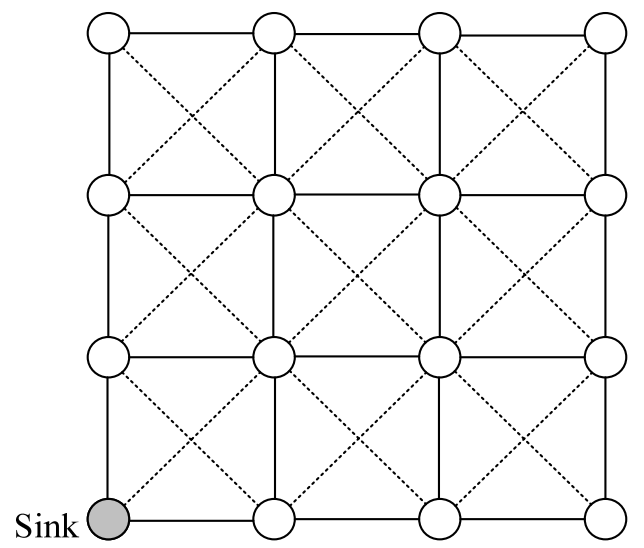

Figure 9. A gird-based topology of WSN in AREA-MAC.
Table 1. Comparing performance of real-time MAC protocols for wireless sensor network.

\begin{tabular}{ccccc}
\hline Protocol & $\begin{array}{c}\text { Energy } \\
\text { efficiency }\end{array}$ & Synchrony & $\begin{array}{c}\text { Timeli- } \\
\text { ness }\end{array}$ & $\begin{array}{c}\text { Adapta- } \\
\text { bility }\end{array}$ \\
\hline $\begin{array}{c}\text { Dual-mode } \\
\text { protocol }\end{array}$ & No & Yes & Yes & Yes \\
RRMAC & No & Yes & Yes & No \\
VTS & Yes & Yes & Yes & No \\
$\begin{array}{c}\text { LPRT } \\
\text { AREA-MA } \\
\text { C }\end{array}$ & Yes & Yes & Yes & No \\
$\begin{array}{c}\text { A novel } \\
\text { real-time } \\
\text { MAC }\end{array}$ & Yes & Yes & Yes & Yes \\
CR-SLF & No & N/A & Yes & No \\
TOMAC & No & N/A & Yes & No \\
SUPORTS & No & N/A & Yes & Yes \\
\hline
\end{tabular}

protocols proposed for sensor networks. As a discussion about what we mentioned above, this section consists of open issues of current real-time MAC protocols and the future research directions.

The keyword of the real-time computing system is deadline. In a real-time control system the packets which are transmitted from source node should arrive at destination before deadline for guaranteeing the timeliness and validity of the alarm messages in the critical and dangerous environments. In order to avoid impact from packets dropping due to miss deadline, packet transmission needs to be scheduled, in other words, the packets need to be allocated priority for transmission sequence since different transmission conditions. For the restricted transmission time, although all of the protocols mention the timeliness which is shown in Table 1, most of the real-time MAC protocols for sensor networks design the algorithm without messages transmission sequence by deadline, instead they just design the algorithm for decreasing the packet transmission latency from source to destination node. In those real-time control MAC protocol for sensor network, the great challenge is to often consider that whether it is able to react to external events quickly. According to this interpretation, an algorithm is considered to be real-time if it is low latency. The term low latency, however, has a relative meaning and does not capture the main properties of real-time control systems.

The other important issue is energy. Because the battery-power, wireless sensor networks not have a permanent lifetime for monitoring environment. There is not a higher reliability if the battery of some nodes exhaust power, since the link of communication is interrupted. 
Therefore the energy-efficient is an important element for assessing a sensor network real-time MAC protocol whether it adapts monitoring application or not. In wireless sensor networks, there is a more required system than conventional real-time computing system, for prolonging lifetime of wireless sensor networks, the real-time MAC protocols should support an energyefficient algorithm to increase the reliability of the sensor networks. As a trade-off, it may cause a high latency in return for gaining more energy conservation. However, latency is a very important parameter in real-time transmission system for sensor networks. The trade-off between energy conservation and latency is an obvious challenge for wireless sensor networks.

\section{Conclusions and Future Work}

This paper has surveyed the real-time medium access control protocols for wireless sensor networks. We have introduced the characteristic of real-time system and discussed the special requirements of wireless sensor network real-time MAC protocols, showed a classification of the research on the current real-time MAC protocols, described architecture of the protocols and discussed the advantage and disadvantage. In addition, we presented the open issues for current sensor networks real-time MAC protocols in the literature.

In summary, most of the existing wireless sensor network real-time MAC protocols focus on decreasing transmission latency, yet still do not adequately consider all of the requirements of sensor networks. In the future, the key challenge which is meeting timing requirement should be guaranteed while establishing a reasonable trade-off and minimizing overhead packets.

\section{Acknowledgements}

This research was supported by the MKE (The Ministry of Knowledge Economy), Korea, under the ITRC (Information Technology Research Center) support program supervised by the NIPA (National IT Industry Promotion Agency) (NIPA-2010-C1090-1031-0007).

\section{References}

[1] I. F. Akyildiz, W. Su, Y. Sankarasubramaniam and E. Cayirci, "A Survey on Sensor Networks," IEEE Communications Magazine, Vol. 40, No. 8, August 2002, pp. 102-114.

[2] G. Anastasi, M. Conti, M. D. Francesco and A. Passarella, "Energy Conservation in Wireless Sensor Networks: A Survey," Ad Hoc Networks, Vol. 7, No. 3, May 2009, pp. 537-568.

[3] K. Kredoand and P. Mohapatra, "Medium Access Control in Wireless Sensor Networks," Computer Networks, Vol.
51, No. 4, March 2007, pp. 961-994.

[4] W. Ye, J. Heidemann and D. Estrin, "An Energy-Efficient Mac Protocol for Wireless Sensor Networks," Proceedings of the 21st Annual Joint Conference of the IEEE Computer and Communications Societies, New York, 23-27 June 2002, pp. 1567-1576.

[5] W. Ye, J. Heidemann and D. Estrin, "Medium Access Control with Coordinated Adaptive Sleeping for Wireless Sensor Networks," IEEE/ACM Transactions on Networking, Vol. 12, No. 3, Jun. 2004, pp. 493-506.

[6] T. H. Hsu and J. S. Wu, "An Application-Specific Duty Cycle Adjustment MAC Protocol for Energy Conserving over Wireless Sensor Networks," Computer Communications, Vol. 31, No. 17, November 2008, pp. 4081-4088.

[7] P. Lin, C. Qiao and X. Wang, "Medium Access Control with a Dynamic Duty Cycle for Sensor Networks," Proceedings of IEEE Wireless Communications and Networking Conference, Atlanta, 21-25 March 2004, pp. 1534-1539.

[8] R. Yadav, S. Varma and N. Malaviya, "Optimized Medium Access Control for Wireless Sensor Network," International Journal of Computer Science and Network Security, Vol. 8, No. 2, February 2008, pp. 334-338.

[9] T. van Dam and K. Langendoen, "An Adaptive Energy-Efficient MAC Protocol for Wireless Sensor Networks," Proceedings of the 1st International Conference on Embedded Network Sensor System, Los Angeles, 5-7 November 2003, pp. 171-180.

[10] S. H. Yang, H. W. Tseng, E. K. Wu and G. H. Chen, "Utilization Based Duty Cycle Tuning MAC Protocol for Wireless Sensor Networks," Proceedings of Global Telecomminications Conference, St. Louis, 2 December 2005, pp. 3258-3262.

[11] S. Du, A. K. Saha and D. B. Johnson, "RMAC: A Routing-Enhanced Duty-Cycle MAC Protocol for Wireless Sensor Networks," Proceedings of 26th Annual IEEE Conference on. Computer Communications, Anchorage, 6-12 May 2007, pp. 1478-1486.

[12] J. Kim and K. H. Park, "An Energy-Efficient, Transport-Controlled MAC Protocol for Wireless Sensor Networks," Computer Networks, Vol. 53, No. 11, July 2009, pp. 1879-1902.

[13] J. Kim, J. Lim, C. Pelczar and B. Jang, "RRMAC: A Sensor Network MAC for Real Time and Reliable Packet Transmission," Proceedings of International Symposium Consumer Electronics, Vilamoura, 14-16 April 2008, pp. $1-4$.

[14] T. Watteyne, I. Augé-Blum and S. Ubéda, "Dual-Mode Real-Time MAC Protocol for Wireless Sensor Networks: A Validation/Simulation Approach," Proceedings of the 1st International Conference on Integrated As Hoc and Sensor Network, Nice, 30-31 May 2006.

[15] T. Watteyne and I. Augé-Blum, "Proposition of a Hard Real-Time MAC Protocol for Wireless Sensor Networks," Proceedings of the 13th IEEE International Symposium on Modeling, Analysis and Simulation of Computer and Telecommunication System, 27-29 September 2005, pp. 533-536. 
[16] A. Krohn, M. Beigl, C, Decker and T. Zimmer, "TOMACReal-Time Message Ordering in Wireless Sensor Networks Using the MAC Layer," Proceedings of 2nd International Workshop on Networked Sensing Systems, San Diego, 27-28 June 2005.

[17] K. Karenos and V. Kalogeraki, "Real-Time Traffic Management in Sensor Networks," Proceedings of IEEE International Real-Time System Symposium, Rio de Janeiro, 5-8 December 2006, pp. 422-434.

[18] E. Egea-López, J. Vales-Alonso, A. S. Martínez-Sala, J. García-Haro, P. Pavón-Mariño and M. V. B. Delgado, "A Wireless Sensor Networks MAC Protocol for Real- Time Applications," Personal and Ubiquitous Computing, Vol. 12, No. 2, February 2008, pp. 111-122.

[19] B. K. Singh and K. E. Tepe, "A Novel Real-Time MAC Layer Protocol for Wireless Sensor Network Applications," Proceedings of IEEE International Conference on
Electro/Information Technogy, Windsor, 7-9 June 2009, pp. 338-343.

[20] H. Li, P. Shenoy and K. Ramamritham, "Scheduling Messages with Deadlines in Multi-Hop Real-Time Sensor Networks," Proceedings of IEEE Real Time and Embedded Technology and Applications Symposium, 7-10 March 2005, pp. 415-425.

[21] J. A. Afonso, L. A. Rocha, H. R. Silva and J. H. Correia, "MAC Protocol for Low-Power Real-Time Wireless Sensing and Actuation," Proceedings of the 11th IEEE International Conference on Electronics, Circuits and Systems, Nice, 10-13 December 2006, pp. 1248-1251.

[22] P. Kumar, M. Gunes, Q. Mushtaq and B. Blywis, "A Real-Time and Energy-Efficient MAC Protocol for Wireless Sensor Networks," Proceedings of the 6th IEEE and IFIP International Conference on Wireless and Optical Communications Networks, 28-30 April 2009, pp. 1-5. 